\title{
Complex diseases and co-morbidities: polycystic ovary syndrome and type 2 diabetes mellitus
}

\author{
Raymond J Rodgers', Jodie C Avery², Vivienne M Moore², Michael J Davies', Ricardo Azziz ${ }^{3}$, Elisabet Stener-Victorin ${ }^{4}$ \\ Lisa J Moran', Sarah A Robertson ${ }^{1}$, Nigel K Stepto ${ }^{5}$, Robert J Norman ${ }^{1,6}$ and Helena J Teede ${ }^{7}$ \\ ${ }^{1}$ Robinson Research Institute, School of Medicine, The University of Adelaide, Adelaide, South Australia, Australia \\ ${ }^{2}$ Robinson Research Institute, School of Public Health, The University of Adelaide, Adelaide, South Australia, Australia \\ ${ }^{3}$ Department of Obstetrics and Gynecology, Medical College of Georgia, Augusta University, Augusta, Georgia, USA \\ ${ }^{4}$ Department of Physiology and Pharmacology Karolinska Institutet, Stockholm, Sweden \\ ${ }^{5}$ Institute for Health and Sport, Victoria University, Melbourne, Australia \\ ${ }^{6}$ FertilitySA, Adelaide, South Australia, Australia \\ ${ }^{7}$ Monash Centre for Health Research and Implementation (MCHRI), School of Public Health and Preventive Medicine, Monash University, Clayton, \\ Victoria, Australia
}

Correspondence should be addressed to R J Rodgers: ray.rodgers@adelaide.edu.au

\begin{abstract}
Objective: Many complex diseases exhibit co-morbidities often requiring management by more than one health specialist. We examined cross-speciality issues that ultimately affect the health and wellbeing of patients with polycystic ovary syndrome (PCOS). PCOS was originally described as a reproductive condition but is now recognised to also be a metabolic and psychological condition affecting $8-13 \%$ of women of reproductive age. With a four-fold increased risk of type 2 diabetes (DM2), the Population Attributable Risk of DM2 that could be avoided if PCOS were eliminated is a substantial $19-28 \%$ of women of reproductive age. To determine the extent to which PCOS is an important consideration in diabetes development, we examined publications, funding, guidelines and predictors of risk of developing DM2.

Results: We found that the topic of PCOS appeared in specialist diabetes journals at only $10 \%$ the rate seen in endocrinology journals - about 1 in 500 articles. We found research funding to be substantially less than for diabetes and found that diabetes guidelines and predictive tools for DM2 risk mostly ignore PCOS. This is surprising since insulin resistance in women with PCOS has a different aetiology and additionally women with PCOS are at increased risk of becoming overweight or obese - high risk factors for DM2. Conclusions: We consider the causes of these concerning anomalies and discuss current activities to address the co-morbidities of PCOS, including the recent development of international guidelines, an international PCOS awareness program and potentially changing the name of PCOS to better reflect its metabolic consequences.
\end{abstract} Key Words

- diabetes - PCOS

\section{Introduction}

Following the recent release of international guidelines for management of polycystic ovary syndrome (PCOS) (1) endorsed by 38 international organisations in 71 countries, the research, clinical and consumer PCOS communities have highlighted the challenges caused by the poor recognition of the metabolic features of PCOS.
To meet these challenges international multifaceted, multilingual education and translation/awareness programs are underway with free resources to be found at https://www.monash.edu/medicine/sphpm/ mchri/pcos. Here, we sought to identify and analyse some of the other problems that need to be addressed https://ec.bioscientifica.com

https://doi.org/10.1530/EC-18-0502 (c) 2019 The authors Published by Bioscientifica Ltd

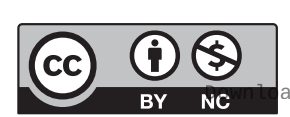

This work is licensed under a Creative Commons Attribution-NonCommercial 4.0 International License. ded from Bioscientifica.com at 04/26/2023 10:36:44AM 
to overcome the poor recognition of the metabolic features of PCOS.

PCOS is a chronic complex disorder that affects more than one in ten women, significantly impacting health and well-being $(2,3)$. The pathophysiology of PCOS is poorly understood and a US National Institute of Health (NIH) evidence-based consensus workshop has recognised that awareness of the condition is limited, while PCOS research is inadequate and poorly funded (4). PCOS is a reproductive and metabolic condition with psychological consequences (5). Women with PCOS suffer symptoms of excess androgen (hirsutism, acne, central adiposity), reproductive dysfunction (infertility, menstrual irregularity, miscarriage, pregnancy complications) and metabolic complications $(6,7)$. Metabolic features include insulin resistance (IR), compensatory hyperinsulinaemia and associated risk of gestational diabetes (GDM), impaired glucose tolerance, type 2 diabetes (DM2), non-alcoholic fatty liver disease (NAFLD), dyslipidaemia and increased risk factors for both cerebrovascular and cardiovascular disease $(6,7,8,9)$. Ultimately, anxiety, depression and distress are increased and quality of life is reduced $(10,11)$. As such, the syndrome is a significant cause of physical and emotional distress to sufferers and is a significant economic burden to individuals and governments (12).

Based on the Rotterdam criteria, the prevalence of PCOS in women of reproductive age is $8-13 \%(3,13)$. Women with PCOS have a four-fold increased risk of DM2 irrespective of their BMI and with a younger disease onset $(14,15)$. Using thesefigures, wecalculate that the Population Attributable Risk (PAR=proportion exposed $\times($ relative risk -1$) / 1+$ proportion exposed $\times($ relative risk -1$)$ ) is $19.4 \%(\mathrm{PAR}=0.08(4-1) /(1+0.08(4-1)))$ to $28 \%(\mathrm{PAR}=0.13$ $(4-1) /(1+0.13(4-1)))$. Thus, the prevalence of DM2 in women of reproductive age would be $19-28 \%$ lower if PCOS could be eliminated. GDM is also increased 2-3-fold in PCOS (16), yet, risk prediction tools for both GDM and DM2 have traditionally not accounted for PCOS.

The true underpinnings of increased risk of DM2 in PCOS remain complex and somewhat unclear. On WHO criteria for IR (clamp-derived glucose infusion rate levels as less than the 25th centile of lean matched controls), a study in 73 women using gold standard clamp techniques showed that $75 \%$ of lean and $95 \%$ of obese women had IR compared to weight-matched controls (17). This was supported by a meta-analysis of clamp studies in PCOS where IR is clearly increased independently of BMI, yet, it is exacerbated by obesity (18). Inherent IR mechanisms in PCOS also appear to differ from those associated with DM2 or obesity $(6,19)$. Insulin-mediated glucose uptake in skeletal muscle and adipose tissue is affected by a post receptor defect in insulin signalling in PCOS (19), that is the subject of ongoing research.

With IR, GDM and DM2 being such major features of PCOS, the link between PCOS, diabetes and metabolic dysfunction appears to be under-appreciated. To explore this further, we analysed some of the barriers that have confronted clinical and basic research into PCOS and highlight some of the recent initiatives to address these issues.

\section{Publications on PCOS}

We first examined journals devoted to the topic of diabetes. We performed PubMed (https://www.ncbi. nlm.nih.gov/pubmed) searches on the terms 'polycystic' OR 'PCOS' in diabetes journals selected from the top 20 ranked journals by impact factor in Thompsons ISI's category of 'Endocrinology and Metabolism' with the word 'diabetes' in the journal name (Table 1). We could find 0, 34, 36, 15 and 4 articles in total in Lancet Diabetes Endocrinology, Diabetes, Diabetes Care, Diabetologia and Diabetes Obesity Metabolism, respectively, at any time prior to the study end date of March, 2017. This equates to around one in every 500 articles addressing the topic of PCOS, which is more than 10-fold lower than in journals devoted to clinical endocrinology or clinical reproduction (Table 1). This is despite 6853 total articles on PCOS addressing insulin, IR or T2DM (searching on (polycystic ovary OR PCOS) AND (insulin OR diabetes) in PubMed). In summary, key relevant diabetes journals publish relatively little on this common condition affecting one in ten women and imparting a high risk of DM2 at a younger age.

Why is PCOS under-represented in diabetes journals? Without knowing the rates of submission and acceptance for relevant journals, it is not possible to assess where the challenges lie, and whether there may be a potential bias in handling of PCOS articles or if authors preferred other journals focused on endocrinology or reproduction. It might be a combination of both as of 'the 8600 papers submitted to Diabetologia since 1 Jan 2013, only 17 articles had PCOS/polycystic in the title (i.e. $0.2 \%$ ), suggesting that one of the main reasons for low penetrance of PCOS in the diabetes journals is that they are not being submitted very much' (Editor of Diabetologia, unpublished observation). Poor funding of PCOS research has probably also led to fewer studies being published on PCOS (20) or perhaps studies have not been conducted to the standard required in diabetes journals. In any event, far fewer articles on 
Table 1 The numbers of articles on PCOS, the total number of articles published and the proportion of articles on PCOS.

\begin{tabular}{|c|c|c|c|}
\hline Journal & Pcos & Total & Pcos/total as \% \\
\hline \multicolumn{4}{|l|}{ Diabetes journals } \\
\hline Lancet Diabetes and Endocrinology & 0 & 924 & 0.0 \\
\hline Diabetes Care & 36 & 15,867 & 0.23 \\
\hline Diabetes & 34 & 15,713 & 0.22 \\
\hline Diabetologia & 15 & 11,062 & 0.14 \\
\hline Diabetes Obesity Metabolism & 4 & 2465 & 0.16 \\
\hline \multicolumn{4}{|l|}{ Clinical endocrinology journals } \\
\hline Journal of Clinical Endocrinology and Metabolism & 866 & 32,867 & 2.63 \\
\hline European Journal of Endocrinology & 162 & 5484 & 3.0 \\
\hline Endocrine & 71 & 3517 & 2.0 \\
\hline \multicolumn{4}{|l|}{ Basic endocrinology journals } \\
\hline Journal of Endocrinology & 61 & 15,042 & 0.40 \\
\hline Endocrinology & 98 & 37,317 & 0.26 \\
\hline \multicolumn{4}{|l|}{ Clinical reproduction journals } \\
\hline Fertility and Sterility & 1288 & 23,528 & 5.47 \\
\hline Human Reproduction & 732 & 13,935 & 5.3 \\
\hline \multicolumn{4}{|l|}{ Basic reproduction journals } \\
\hline Reproduction & 4 & 2853 & 0.1 \\
\hline Biology of Reproduction & 66 & 13,807 & 0.5 \\
\hline
\end{tabular}

The data were collected in early 2017 and the search terms used were 'PCOS' and 'polycystic', and both the titles and abstracts were searched in PubMed. The numbers of articles include articles containing primary data and review articles.

PCOS are published in the diabetes literature than in endocrinology and reproduction journals, and this rate appears to be lower than the high diabetes risk status would warrant.

\section{Research funding of PCOS}

Dr Ricardo Azziz and his colleagues examined funding by the NIH in the USA over 10 years. They identified that PCOS received substantially less funding than other conditions for which the prevalence, economic burden, metabolic morbidity and negative impact on quality of life was much less than PCOS (20). In Australia the National Health and Medical Research Council (NHMRC) is the premier funder of medical research and its main funding mechanism is by way of project grants with currently over 600 granted per annum. Using NHMRC published data and searching the titles of the funded project grants for the terms 'PCOS' or 'polycystic', there were only nine funded project grants in 16 years (2003-2018) (21). Searching the term 'diabetes' in the titles of project grants for the same period we identified 196 funded grants. These data identify that that PCOS receives $<5 \%$ of the funding that diabetes receives but contributes proportionately more to the prevalence of GDM and DM2.

Anecdotally, colleagues from other countries have found it strategically advantageous not to address PCOS issues when applying for research grants, but rather to examine broader issues that include PCOS as a subgroup.
Additionally, whilst there are philanthropic organisations supporting research into many areas such as diabetes, cancer and cardiovascular diseases, we are unaware of any such foundations for PCOS. This raises the question as to why research into PCOS is so poorly funded. Part of the reason could be that the secondary pathologies associated with the syndrome, like obesity or DM2, take priority over PCOS which is perceived by many health professionals as primarily a reproductive disorder (22).

\section{PCOS diagnosis and guidelines}

PCOS was originally described as a reproductive condition (23), and it was not recognised that these patients had perturbed insulin and glucose handling until the 1980s (24). Additionally PCOS is multifactorial and there have been two major consensus meetings to define its diagnostic criteria referred to as the National Institute of Health (NIH) criteria (original) and the Rotterdam criteria (now NIH endorsed) (4). The first evidence-based guidelines in PCOS were produced in Australia in 2011 (10) and international co-funded rigorous evidence-based guidelines have only recently been published (1).

The Rotterdam criteria stipulate two of the following symptoms (1) polycystic ovaries, (2) evidence of androgen excess and (3) oligo- or amenorrhea. These criteria were recently internationally endorsed (25) and are founded in evidence with the international evidence-based guideline for the assessment and management of PCOS

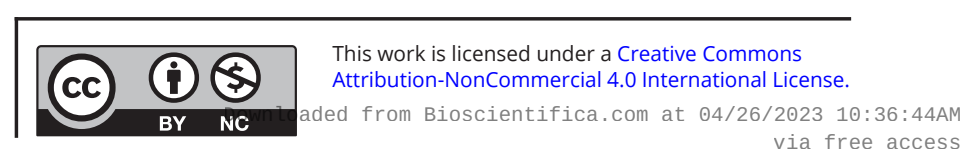


in adults. These criteria are supported by recent genome wide association data showing common genotypes across all diagnostic criteria (26). In women within 8 years of menarche, both (1) irregular cycles and (2) evidence of androgen excess are needed for diagnosis, whilst ultrasound detection of polycystic ovarian morphology was not recommended in diagnosis due to inaccuracy in this group (1).

PCOS has been recognised as a non-modifiable risk factor for diabetes, yet often PCOS was not considered in predictive tools for diabetes (27). However, some more recent predictive tools are taking PCOS into account (28). The research, clinical and consumer PCOS communities have highlighted the challenge that the metabolic features of PCOS are poorly recognised and an international PCOS awareness program is now established. Currently international multifaceted, multilingual education and translation/awareness programs are underway with free resources to be found at https://www.monash.edu/ medicine/sphpm/mchri/pcos.

\section{Changing the name of PCOS}

The name of PCOS does not indicate the full breadth of complications. Additionally, the term 'polycystic ovary' is a misnomer, and it is not necessary or sufficient to describe PCOS. A recent survey interviewed 105 clinicians and 57 women diagnosed with PCOS to understand perceptions about the key clinical features of the syndrome (29). The majority of women and clinicians $(86 \%, 90 \%)$ identified 'irregular periods' as a key feature, with 'hormonal imbalance' $(60 \%)$ as the next most popular. Incorrectly $47 \%$ of women identified 'ovarian cysts' as the key feature. Almost half of the women (48\%) found the name to be confusing (29).

In regard to the name, a NIH PCOS evidence-based workshop (4) stated 'We believe the name 'PCOS' is a distraction and an impediment to progress. It causes confusion and is a barrier to effective education of clinicians and communication with the public and research funders. The name focuses on a criterion polycystic ovarian morphology - which is neither necessary nor sufficient to diagnose the syndrome. We believe it is time to recognise the advances that have been made since the description of the syndrome by Irving F Stein Sr and Michael L Leventhal. It is time to expeditiously assign a name that reflects the complex metabolic, hypothalamic, pituitary, ovarian and adrenal interactions that characterise the syndrome and their reproductive implications. The right name will enhance recognition of this major public health issue for women, educational outreach, 'branding' and public relations and will assist in expanding research support. There is now an international dialogue to drive a name change with a view to increasing understanding of the prevalence and its diverse key features, including DM2 (30).

\section{Conclusions}

Based upon the prevalence of PCOS and its seriously high risk factors for IR, GDM and DM2, it is clear that PCOS has not received the attention it warrants, especially in the diabetes arena. One wonders if the metabolic phenotypes of PCOS had been recognised from the outset and if the syndrome had not been named incorrectly or better named to reflect its metabolic phenotype, PCOS might have been a higher funding and publishing priority. It is not possible from our analyses to discern if the low emphasis on PCOS within the diabetes arena is due to specific issues around PCOS or if this is simply an issue around complex diseases requiring multi-disciplinary approaches wherein one discipline rates the disease less highly than another. Going forward it will be informative to track how the mental health arena regards PCOS now that it has been recognised as a risk factor for issues involving mental health and wellbeing. In the meantime, we advocate that PCOS needs to be better recognised, including by the diabetes community, for its substantial and unique metabolic features and its important contribution to the development of DM2.

\section{Declaration of interest}

The authors declare that there is no conflict of interest that could be perceived as prejudicing the impartiality of this review.

\section{Funding}

This work did not receive any specific grant from any funding agency in the public, commercial or not-for-profit sector.

\section{Acknowledgements}

The authors thank the NHMRC for supporting the Centre for Research Excellence in the evaluation, management and health care needs of Polycystic Ovary Syndrome and related health implications. This centre has fostered much of what has been presented here.

\section{References}

1 Teede HJ, Misso ML, Costello MF, Dokras A, Laven J, Moran L, Piltonen T, Norman RJ \& International PCOS Network. Recommendations from the international evidence-based guideline for the assessment and management of polycystic ovary syndrome.

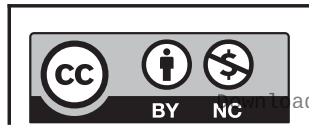

This work is licensed under a Creative Commons Attribution-NonCommercial 4.0 International License. ded from Bioscientifica com at 04/26/2023 10:36:44AM 
Clinical Endocrinology 201889 251-268. (https://doi.org/10.1111/ cen.13795)

2 March WA, Moore VM, Willson KJ, Phillips DI, Norman RJ \& Davies MJ. The prevalence of polycystic ovary syndrome in a community sample assessed under contrasting diagnostic criteria. Human Reproduction 2010 25 544-551. (https://doi.org/10.1093/humrep/dep399)

3 Bozdag G, Mumusoglu S, Zengin D, Karabulut E \& Yildiz BO. The prevalence and phenotypic features of polycystic ovary syndrome: a systematic review and meta-analysis. Human Reproduction $2016 \mathbf{3 1}$ 2841-2855. (https://doi.org/10.1093/humrep/dew218)

4 National Institutes of Health. Evidence-based methodology workshop on polycystic ovary syndrome: executive summary. Bethesda, MD, USA: NIH, 2012. (available at: https://prevention.nih. gov/sites/default/files/2018-06/FinalReport.pdf)

5 Teede H, Deeks A \& Moran L. Polycystic ovary syndrome: a complex condition with psychological, reproductive and metabolic manifestations that impacts on health across the lifespan. BMC Medicine 20108 41. (https://doi.org/10.1186/1741-7015-8-41)

6 Azziz R, Carmina E, Chen Z, Dunaif A, Laven JS, Legro RS, Lizneva D, Natterson-Horowtiz B, Teede HJ \& Yildiz BO. Polycystic ovary syndrome. Nature Reviews Disease Primers 20162 16057. (https://doi. org/10.1038/nrdp.2016.57)

7 Norman RJ, Dewailly D, Legro RS \& Hickey TE. Polycystic ovary syndrome. Lancet $2007 \mathbf{3 7 0}$ 685-697. (https://doi.org/10.1016/ S0140-6736(07)61345-2)

8 Padmanabhan V. Polycystic ovary syndrome - 'a riddle wrapped in a mystery inside an enigma'. Journal of Clinical Endocrinology and Metabolism 200994 1883-1885. (https://doi.org/10.1210/jc.2009-0492)

9 Diamanti-Kandarakis E, Kandarakis H \& Legro RS. The role of genes and environment in the etiology of PCOS. Endocrine 200630 19-26. (https://doi.org/10.1385/ENDO:30:1:19)

10 Teede HJ, Misso ML, Deeks AA, Moran LJ, Stuckey BG, Wong JL, Norman RJ, Costello MF \& Guideline Development Groups. Assessment and management of polycystic ovary syndrome: summary of an evidence-based guideline. Medical Journal of Australia 2011195 S65-S112. (https://doi.org/10.5694/mja11.10915)

11 Cooney LG, Lee I, Sammel MD \& Dokras A. High prevalence of moderate and severe depressive and anxiety symptoms in polycystic ovary syndrome: a systematic review and meta-analysis. Human Reproduction 201732 1075-1091. (https://doi.org/10.1093/humrep/ dex044)

12 Azziz R, Marin C, Hoq L, Badamgarav E \& Song P. Health care-related economic burden of the polycystic ovary syndrome during the reproductive life span. Journal of Clinical Endocrinology and Metabolism 200590 4650-4658. (https://doi.org/10.1210/jc.2005-0628)

13 March WA, Moore VM, Willson KJ, Phillips DI, Norman RJ \& Davies MJ. The prevalence of polycystic ovary syndrome in a community sample assessed under contrasting diagnostic criteria. Human Reproduction 2010 25 544-551. (https://doi.org/10.1093/humrep/dep399)

14 Moran LJ, Misso ML, Wild RA \& Norman RJ. Impaired glucose tolerance, type 2 diabetes and metabolic syndrome in polycystic ovary syndrome: a systematic review and meta-analysis. Human Reproduction Update 201016 347-363. (https://doi.org/10.1093/humupd/dmq001)

15 Moran LJ, Strauss BJ \& Teede HJ. Diabetes risk score in the diagnostic categories of polycystic ovary syndrome. Fertility and Sterility 201195 1742-1748. (https://doi.org/10.1016/j.fertnstert.2011.01.133)

16 Joham AE, Ranasinha S, Zoungas S, Moran L \& Teede HJ. Gestational diabetes and type 2 diabetes in reproductive-aged women with polycystic ovary syndrome. Journal of Clinical Endocrinology and Metabolism 201499 E447-E452. (https://doi.org/10.1210/jc.2013-2007)
17 Stepto NK, Cassar S, Joham AE, Hutchison SK, Harrison CL, Goldstein RF \& Teede HJ. Women with polycystic ovary syndrome have intrinsic insulin resistance on euglycaemic-hyperinsulaemic clamp. Human Reproduction 201328 777-784. (https://doi. org/10.1093/humrep/des463)

18 Cassar S, Misso ML, Hopkins WG, Shaw CS, Teede HJ \& Stepto NK. Insulin resistance in polycystic ovary syndrome: a systematic review and meta-analysis of euglycaemic-hyperinsulinaemic clamp studies. Human Reproduction 201631 2619-2631. (https://doi.org/10.1093/ humrep/dew243)

19 Diamanti-Kandarakis E \& Dunaif A. Insulin resistance and the polycystic ovary syndrome revisited: an update on mechanisms and implications. Endocrine Reviews 201233 981-1030. (https://doi. org/10.1210/er.2011-1034)

20 Brakta S, Lizneva D, Mykhalchenko K, Imam A, Walker W, Diamond MP \& Azziz R. Perspectives on polycystic ovary syndrome: is polycystic ovary syndrome research underfunded? Journal of Clinical Endocrinology and Metabolism 2017102 4421-4427. (https:// doi.org/10.1210/jc.2017-01415)

21 Rodgers RJ, Avery JC \& McAllister V. Commentary: a new evidencebased guideline for assessment and management of polycystic ovary syndrome. Medical Journal of Australia 2019.

22 Dokras A, Saini S, Gibson-Helm M, Schulkin J, Cooney L \& Teede H. Gaps in knowledge among physicians regarding diagnostic criteria and management of polycystic ovary syndrome. Fertility and Sterility 2017 107 1380.e1-1386.e1. (https://doi.org/10.1016/j.fertnstert.2017.04.011)

23 Stein IF \& Leventhal ML. Amenorrhea associated with bilateral polycystic ovaries. American Journal of Obstetrics and Gynecology 1935 29 181-191. (https://doi.org/10.1016/S0002-9378(15)30642-6)

24 Burghen GA, Givens JR \& Kitabchi AE. Correlation of hyperandrogenism with hyperinsulinism in polycystic ovarian disease. Journal of Clinical Endocrinology and Metabolism $1980 \mathbf{5 0}$ 113-116. (https://doi.org/10.1210/jcem-50-1-113)

25 Teede HJ, Misso ML, Costello MF, Dokras A, Laven J, Moran L, Piltonen T, Norman RJ \& International PCOS Network. Recommendations from the international evidence-based guideline for the assessment and management of polycystic ovary syndrome. Fertility and Sterility $2018110364-379$. (https://doi.org/10.1016/j. fertnstert.2018.05.004)

26 Day F, Karaderi T, Jones MR, Meun C, He C, Drong A, Kraft P, Lin N, Huang $\mathrm{H}$, Broer L, et al. Large-scale genome-wide meta-analysis of polycystic ovary syndrome suggests shared genetic architecture for different diagnosis criteria. PLoS Genetics 201814 e1007813. (https:// doi.org/10.1371/journal.pgen.1007813)

27 Abdul-Ghani MA, Abdul-Ghani T, Stern MP, Karavic J, Tuomi T, Bo I, Defronzo RA \& Groop L. Two-step approach for the prediction of future type 2 diabetes risk. Diabetes Care $2011342108-2112$. (https://doi.org/10.2337/dc10-2201)

28 Capula C, Chiefari E, Borelli M, Oliverio R, Vero A, Foti D, Puccio L, Vero R \& Brunetti A. A new predictive tool for the early risk assessment of gestational diabetes mellitus. Primary Care Diabetes 201610 315-323. (https://doi.org/10.1016/j.pcd.2016.05.004)

29 Teede H, Gibson-Helm M, Norman RJ \& Boyle J. Polycystic ovary syndrome: perceptions and attitudes of women and primary health care physicians on features of PCOS and renaming the syndrome. Journal of Clinical Endocrinology and Metabolism 201499 E107-E111. (https://doi.org/10.1210/jc.2013-2978)

30 Azziz R. Polycystic ovary syndrome: what's in a name? Journal of Clinical Endocrinology and Metabolism 201499 1142-1145. (https:// doi.org/10.1210/jc.2013-3996)

Received in final form 4 February 2019

Accepted 13 February 2019

Accepted Preprint published online 13 February 2019 https://ec.bioscientifica.com https://doi.org/10.1530/EC-18-0502 (c) 2019 The authors Published by Bioscientifica Ltd

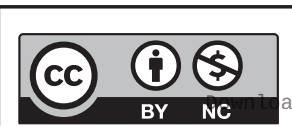

This work is licensed under a Creative Commons Attribution-NonCommercial 4.0 International License. ded from Bioscientifica com at 04/26/2023 10:36:44AM 